\title{
Especialización y productividad del sector servicios en Nayarit, 1980-2003
}

\section{Specialization and productivity of the services sector in Nayarit, 1980-2003}

\author{
Karla Susana Barrón-Arreola \\ Jesús Antonio Madera-Pacheco*
}

\begin{abstract}
In recent decades the importance of the urbanization process and the growing relevance of the services sector inside economies have been the object of analysis from different disciplinary and methodological approaches. Nevertheless, for the case of Nayarit, few have analyzed the behavior and evolution of the productive structure, especially of its services sector. We intend to contribute with this work, analyzing the effect of specialization and productivity of the services sector in the economic growth of the State of Nayarit during the period 1980-2003.
\end{abstract}

Keywords: services sector; Verdoorn's coefficient; returns to scale.

\section{Resumen}

En las últimas décadas, la importancia de los procesos de urbanización y la creciente relevancia del sector servicios al interior de las economías, han sido motivo de análisis desde diferentes enfoques disciplinarios y metodológicos. No obstante, para el caso de Nayarit, muy pocos estudios han analizado el comportamiento y evolución de la estructura productiva, en especial, de su sector terciario. A ello se pretende contribuir con este trabajo, analizando el efecto de la especialización y la productividad del sector servicios en el crecimiento económico del estado de Nayarit durante el periodo 1980-2003.

Palabras clave: servicios, coeficiente de Verdoorn, rendimientos a escala. 


\section{Introducción $^{1}$}

El crecimiento económico ha sido tema de estudio en los niveles sectorial y regional, estudios donde además se ha incorporado la relación de éste con los patrones de especialización. Independientemente del enfoque teórico con que se aborden, tales patrones se pueden clasificar con base en el mecanismo que les permita especializarse, pueden ser de carácter smithiano, es decir, derivado del aprendizaje por experiencia y donde el tipo de bienes que generan rendimientos crecientes no resulta tan relevante; o bien, de carácter ricardiano, donde el proceso de especialización es resultado de las ventajas comparativas, debido a la producción de bienes con mayores oportunidades tecnológicas.

Por otra parte, en las últimas décadas la importancia de los procesos de urbanización y la creciente relevancia del sector servicios al interior de las economías, han sido motivo de análisis desde diferentes enfoques disciplinarios y metodológicos, entre ellos, los desarrollados por Garza (2005, 2006, 2008, entre otros). En el caso de Nayarit, muy pocos estudios han analizado el comportamiento y evolución de la estructura productiva, en especial de su sector terciario. A ello se pretende contribuir con este trabajo, analizando el efecto de la especialización y la productividad del sector servicios en el crecimiento económico del estado de Nayarit durante el periodo $1980-2003 .^{2}$

La estructura del trabajo presenta en la primera parte una breve revisión de los procesos de especialización, vista desde los modelos de crecimiento económico; la relación entre productividad y crecimiento económico se analiza en la parte segunda, en tanto que el tercer apartado incluye un análisis de la economía de Nayarit y del sector servicios. La metodología utilizada para el análisis de la especialización y productividad de este sector en Nayarit, se aborda en la cuarta parte, mientras que la evidencia empírica de dicho análisis aparece en la parte quinta. Finalmente, se presentan algunas reflexiones a manera de conclusión.

\section{Crecimiento económico y especialización}

Una de las tareas de los estudiosos de la economía, desde tiempos de Adam Smith y David Ricardo, ha sido responder a interrogantes como: ¿̨por qué

\footnotetext{
${ }^{1}$ Los autores reconocemos los atinados comentarios y sugerencias realizados por los dos evaluadores anónimos para mejorar este escrito. Este documento es parte del proyecto "Productividad, especialización y crecimiento económico en México: una visión postkeynesiana”, con el financiamiento de Promep, 2007.

${ }^{2}$ El periodo de análisis se definió por la disponibilidad de información proporcionada por el INEGI, en particular la correspondiente a comercio y servicios. Sin embargo, a fin de contextualizar la economía nayarita, en algunos casos se utiliza información de 1970 a 2006.
} 
algunas regiones crecen más que otras?, ¿por qué se especializan en la producción de determinados productos?, ¿qué determina los patrones de especialización en cada región?, ¿̇tales patrones son un factor importante para el crecimiento económico?

Desde la visión de Smith (1984), la especialización es el resultado del aprendizaje asociado a la experiencia, que permitía aumentar la productividad del trabajo y la obtención de rendimientos crecientes sin importar el tipo de bienes que se produjeran. Es decir, si una región podía ser más eficiente que otra en la producción de algunos bienes y menos eficiente en la producción de otros, independientemente de la causa de la diferencia en la eficiencia, ambas regiones se podrían beneficiar si cada una se especializa en lo que puede ser más eficiente (Chacholiades, 1992). Se trata, pues, de un modelo basado en las ventajas absolutas que, a su vez, son determinadas por los beneficios de la especialización, donde ambas regiones pueden acceder a otros bienes mediante intercambios comerciales (Tugores, 2002).

A diferencia de un modelo como el planteado por Smith, para Ricardo la especialización es el resultado de ventajas comparativas, que se obtienen al orientar la producción a áreas y bienes que tengan las mejores oportunidades tecnológicas y/o de ventas en otros mercados. Así, la ventaja comparativa de una región estará determinada por aquel bien cuyo grado de superioridad (entendido como productividad) sea mayor, en tanto que en el bien que tenga un grado menor de superioridad, en relación con otra región, representará una desventaja comparativa (Chacholiades, 1992).

De manera sintética, la diferencia entre las ventajas absolutas y las comparativas es que, en la primera, la producción de un bien corresponde a una mayor productividad, en tanto que en la segunda dependerá del bien en que la ventaja de productividad es mayor (Tugores, 2002). En este mismo sentido, Jungmittag (2004) establece que las ventajas absolutas están asociadas al learning-by-doing y a los rendimientos crecientes a escala, y que no dependen de las áreas en las que esté especializada la región: la llama visión Smithiana. Por su parte, las ventajas comparativas se asocian al aspecto cualitativo de la especialización vinculado a las economías de escala y la dotación de factores en los sectores (Laursen, 1998). Ambos planteamientos se pueden identificar en modelos de crecimiento económico que buscan abonar en el análisis sobre especialización.

Desde el planteamiento smithiano, el grado de especialización estará determinado por la menor dispersión de actividades en las empresas o regiones. En los modelos de la nueva teoría del crecimiento se encuentra el que plantea Romer (1986) que, en un contexto neoclásico, logra introducir los conceptos de learnig-by-doing y aprendizaje, donde el progreso 
técnico es de carácter endógeno y la acumulación de conocimientos (derivado de la investigación realizada por las empresas en la búsqueda de maximizar beneficios) son el motor de crecimiento. En esta visión, el rendimiento social de la investigación es mayor que el individual. ${ }^{3}$

Derivado de la misma corriente teórica destaca también el planteamiento de Lucas (1988), quien mediante un modelo de dos sectores introduce la creación y acumulación de capital humano por medio del learning-by-doing. En este modelo, el primer sector se dedica a la producción final y el segundo a la producción y acumulación de capital humano. ${ }^{4}$ Para ambos sectores, los insumos comprenden capital humano y capital físico. El primero está asociado a rendimientos crecientes a escala, lo que garantiza crecimiento sin límites en la economía.

Es decir, si dos regiones similares producen bienes sustitutos, cada una tenderá a especializarse en bienes que representen una ventaja comparativa con respecto a la otra, permitiendo una completa especialización derivada del aprendizaje. Así, la economía con mayor crecimiento será la que logre especializarse en la producción de bienes de alto contenido tecnológico; sin embargo, el patrón de especialización será determinado por las ventajas comparativas y por la dotación inicial de capital humano (Barrón, 2009).

Un tercer modelo es el que presentan Rivera y Romer (1991), en el cual existen dos tipos de actividades manufactureras: producción de bienes de consumo y producción de bienes de capital (sin innovaciones tecnológicas); además agregan un tercer sector orientado a actividades de investigación y desarrollo (I\&D) que genere nuevos bienes de capital. Los autores concluyen que la difusión de conocimiento es fundamental para lograr la integración económica, ya que es un acelerador del crecimiento no sólo por el flujo de bienes, sino también de ideas. Es decir, en el modelo, lo que determina la integración de dos economías son los rendimientos crecientes a escala en la función de producción, ya que es más eficiente tener sólo un sector dedicado a la producción de un bien (sea de consumo, capital o conocimiento), que tener dos sectores aislados produciendo el mismo tipo de bien y duplicando esfuerzos. En este sentido, Klenow y Rodríguez (2004) señalan que para las regiones será preferible la especialización para inventar nuevos productos que producir lo mismo que en el resto de las regiones.

Por otro lado, en la postura de especialización de carácter ricardiano, se encuentra el planteamiento de Romer (1990), en el que se presentan tres sectores productivos utilizando capital físico, trabajo, capital humano

\footnotetext{
${ }^{3}$ Es decir, el conocimiento generado por las empresas es una externalidad, ya que tiene un efecto positivo en las empresas restantes.

${ }^{4}$ Ésta es proporcional a la fracción de trabajadores dedicados a la producción en cada sector.
} 
y tecnología como insumos. El primer sector se orienta a producir bienes de consumo y los insumos utilizados son trabajo, capital humano y productos de capital para generar producción; el segundo sector produce bienes de capital partiendo de los diseños hechos por el tercer sector; $y$ un tercer sector orientado a la investigación, cuyos insumos son capital humano y stock de conocimiento existente para producir nuevos conocimientos. Desde este planteamiento, a medida que aumenta el stock de diseños y conocimientos, será mayor la productividad de un trabajador en el sector de I\&D, por otro lado, los incentivos que motivan la investigación en nuevos descubrimientos son los ingresos monopólicos que las empresas pueden lograr, al menos por un tiempo, asociados a la existencia de patentes y derechos de propiedad. Lo que destaca en el modelo, de manera implícita, es la importancia de la especialización en sectores donde las oportunidades de innovación son mayores y registran tasas de crecimiento más altas. Este modelo permite entender por qué economías con bajos niveles de capital humano y poca apertura comercial, han tenido un mayor rezago, es decir, una economía con un amplio stock de capital humano, experimentará tasas de crecimiento más rápidas, sugiriendo además que el crecimiento económico lo puede acelerar el comercio internacional.

El trabajo de Grossman y Helpman (1991) incluye supuestos que incorporan el incremento del número de bienes en el mercado, tanto por la mejora en la calidad de los productos predecesores como por un aumento en la variedad de bienes disponibles en la economía. En el modelo, el comercio tiene efecto en la tasa de crecimiento de un país, derivado del intercambio de ideas, que aumentarán globalmente el stock de conocimientos, lo que reduce la inversión necesaria para futura I\&D en cada economía o región. Si ambas regiones tienen la misma tasa de innovación, se evitará la duplicidad de recursos dedicados a la investigación mediante el comercio, pudiendo acceder a otros mercados y bienes. En el caso de tasas de innovación diferente, la región que tenga la más alta incrementará su cuota de mercado por la generación de nuevos productos, en tanto que en la de menor grado el efecto será negativo.

Los modelos de Romer (1990) y de Grossman y Helpman (1991) tienen como rasgo común el carácter cualitativo de la especialización de un país, ya que esto le permitirá obtener tasas de productividad mayores, lo que se traducirá en una mejor posición para obtener una tasa de crecimiento más rápida. Sin embargo, las limitaciones de estos modelos radican en que asumen la existencia de un mecanismo de ajuste que vaciará los mercados, así como en la racionalidad de los agentes económicos, lo que resulta inadmisible en un marco donde se permite la existencia de 
rendimientos crecientes a escala y se subraya la importancia del cambio tecnológico (Meliciani, 2001).

Es importante señalar que en la postura ricardiana también se identifican modelos de corte poskeynesiano, donde se destaca el efecto del comercio en el crecimiento económico, tanto por el incremento de la demanda que enfrentan las empresas nacionales como por la contribución que hacen en liberar a la economía de la restricción que impone la balanza de pagos (Meliciani, 2001).

Desde esta corriente, el primer modelo que se identifica es el planteado por Kaldor (1966), en el cual se hace una distinción entre los sectores de la economía, identificando que las actividades agrícolas están asociadas a rendimientos constantes a escala, en tanto que las actividades manufactureras las vincula a rendimientos crecientes a escala. En este modelo se considera la industria manufacturera como el motor del crecimiento, y se demuestra mediante las denominadas Leyes de Kaldor. ${ }^{5}$ Según este planteamiento, el grado de especialización en actividades manufactureras generará externalidades positivas en el resto de los sectores de la región (Ocegueda, 2003a).

Las diferencias en las tasas de crecimiento entre regiones, así como al interior de ellas, las plantea Kaldor (1970), donde la especialización y la división del trabajo se pueden relacionar con la dotación inicial de recursos y donde las ventajas comparativas juegan un papel importante en actividades que requieren un proceso productivo. En este modelo se hace evidente que una estructura productiva relacionada con la industria moderna, será característica de economías actuales donde la expansión de la demanda será la determinante del incremento de inversión en esas actividades, lo que contribuirá a un mayor crecimiento de la región.

Dixon y Thirlwal (1975) presentan un modelo en el cual formalizan los planteamientos de Kaldor (1970), considerando el efecto Verdoorn como el origen de las diferencias de las tasas de crecimiento regional, así como el comportamiento de largo plazo. Partiendo del supuesto de dos regiones aisladas, cada una con dos sectores (agricultura e industria) y con diferentes tasas de crecimiento de equilibrio estable. Los resultados del modelo establecen que para cada una de las regiones la demanda externa será el principal factor de demanda interna. La especialización en la pro-

${ }^{5}$ En la primera ley se seńala que existe una alta correlación entre la tasa de crecimiento del producto de una economía y la tasa de crecimiento del producto manufacturero; la segunda ley retoma el planteamiento de Verdoorn (1949), donde postula que existe una fuerte relación de causalidad positiva entre el crecimiento del producto manufacturero y el aumento de la productividad del sector, derivado de los rendimientos crecientes a escala tanto estáticos como dinámicos; la tercera ley señala la relación de causalidad positiva entre la tasa de crecimiento del sector manufacturero y el crecimiento de la productividad en el sector no manufacturero, asociada a que en actividades tradicionales (agricultura y algunas de servicios) se presentan rendimientos decrecientes a escala. 
ducción favorece el crecimiento económico, siempre y cuando lo haga en bienes con altas elasticidades ingreso, con lo que se eleva la competitividad en el comercio internacional, permitiendo así una apropiación creciente de la demanda externa a medida que se incrementa. ${ }^{6}$

El crecimiento económico y la especialización productiva están directamente relacionados con la estructura de una economía, resaltando la importancia de los sectores que la componen en el sentido de que no todos pueden promover la expansión de otros sectores o la generación de empleos de alta productividad (Cimoli et al., 2006). En relación con ello, Ezcurra et al. (2002) señalan que las regiones que tienen mejores resultados, en términos de renta per cápita, son aquéllas especializadas en sectores dinámicos y en expansión, cuya estructura sectorial se asocia a la existencia de ventajas comparativas relacionadas con su localización; sin embargo, el patrón de especialización no puede determinarse independientemente de las condiciones iniciales y de la historia de las regiones (Ros, 2001).

En este sentido, Velázquez y Andablo (1997) señalan que el crecimiento y desarrollo económico de una región no se debe basar sólo en una sólida estructura productiva, sino que debe estar acompañada de una gama de servicios acorde con el desarrollo de la región, ya que sin la infraestructura provista por los servicios, la agricultura y la industria no podrían existir (Shugan, 1994). De hecho, en las economías actuales, tanto desarrolladas como en vías de desarrollo, el sector servicios ha venido desplazando a sectores tradicionales, como la agricultura y la industria, tanto en materia de número de unidades de producción como en la cantidad de empleos que generan.

\section{Crecimiento económico y productividad}

La productividad del trabajo ha sido abordada desde diferentes posturas teóricas. La corriente poskeynesiana identifica la demanda como factor que restringe el crecimiento económico, y ubica al sector de las manufacturas como motor de la economía, pues genera rendimientos crecientes a escala, asociados a la división del trabajo y la especialización, cuya identificación se hace mediante la estimación de la ley de Verdoorn.

La relación estadística entre el crecimiento de la productividad del trabajo de largo plazo y la tasa de crecimiento del producto, es la deno-

\footnotetext{
${ }^{6}$ Es decir, una ventaja comparativa inicial en la producción de bienes con alta elasticidad ingreso de la demanda, hará que para la otra región sea muy difícil competir en la producción de los mismos bienes.
} 
minada ley de Verdoorn. ${ }^{7}$ McCombie et al. (2002) señalan que la importancia de esta relación es la evidencia de rendimientos crecientes a escala en las empresas, de carácter interno y externo.

Kaldor (1966) establece en su segunda ley, tomando como base el planteamiento de Verdoorn (1949), ${ }^{8}$ que una rápida tasa de crecimiento de la productividad es inducida por un rápido crecimiento del producto, así, sugiere que existe una fuerte relación de causalidad positiva en el crecimiento del producto (de la manufactura) y el crecimiento de la productividad en el sector, como resultado de los rendimientos a escala estáticos y dinámicos.

En este sentido, Katz (1968) señala que en la ley de Verdoorn existe una relación de causalidad, donde existe una mayor división del trabajo en los procesos de producción y una mayor productividad, relacionadas con la existencia de economías de escala de carácter endógeno y exógeno, obtenidas con la expansión del producto. Relación que es aún más fuerte en economías donde el cambio estructural no se ha dado de manera rápida, además de que es base de los modelos de causación acumulativa y donde las economías de escala en el contexto regional juegan un papel importante (León y Thirlwall, 2002).

Aun cuando los estudios realizados con respecto a la ley de Verdoorn destacan la importancia del sector de las manufacturas en el proceso de crecimiento y desarrollo de las economías, la estimación de esta ley se puede hacer extensiva al sector de los servicios, ya que en las economías actuales es posible encontrar más de un sector líder (Paschaloudis y Alexiadis, 2001).

Para México, la aplicación de esta ley demuestra que las actividades no primarias, en especial la manufactura y en menor medida las terciarias, operan con rendimientos crecientes a escala de carácter dinámico (Ocegueda, 2003a). En el caso de Nayarit, Barrón (2009) encuentra que existen rendimientos crecientes a escala en algunas ramas de manufactura y servicios.

\footnotetext{
${ }^{7}$ Esta ley ha sido utilizada en gran número de estudios que proveen evidencia empírica, confirmando los resultados para diferentes regiones y países, entre ellos destaca el trabajo de McCombie y Ridder (1983), donde señalan que la utilización de datos en el ámbito regional constituye una ventaja, ya que existen menores posibilidades de error de especificación que con datos a nivel internacional. Sin embargo, las estimaciones con este tipo de datos arrojarán un coeficiente de Verdoorn con un límite menor al obtenido con datos internacionales, lo que se asocia a que las economías de escala de carácter nacional son capturadas en el intercepto más que en el coeficiente regional de la ley de Verdoorn (McCombie y Ridder, 1984; Bairam, 1987).

${ }^{8}$ Analiza las series históricas del sector industrial para 14 países: encuentra que el valor promedio de la elasticidad de la productividad respecto al producto era de 0.45 , lo que se traduce como que en el largo plazo un cambio en el volumen de la producción de $10 \%$ se relaciona con un incremento de la productividad del trabajo de 4.5 por ciento.
} 


\section{Una panorámica de la estructura económica y del sector servicios de Nayarit}

\section{1. ¿Una servicialización de la economía nayarita?}

En este apartado presentamos un panorama general de la economía nayarita, con base en los principales indicadores macroeconómicos que nos permitirán mostrar no sólo su tendencia durante las últimas décadas, sino también identificar algunas de sus fortalezas y debilidades.

Situado entre las montańas y el mar, en la vertiente occidental de la Sierra Madre se encuentra el territorio que conforma al estado de Nayarit, cuyo dinamismo económico se explica en gran parte por las actividades agropecuarias, así como por las del sector servicios. Política y administrativamente, el estado de Nayarit se divide en 20 municipios: Acaponeta, Ahuacatlán, Amatlán de Cañas, Compostela, Huajicori, Ixtlán del Río, Jala, Xalisco, El Nayar, Rosamorada, Ruiz, San Blas, San Pedro Lagunillas, Santa María del Oro, Santiago Ixcuintla, Tecuala, Tepic, Tuxpan, La Yesca y Bahía de Banderas.?

Casi de manera tradicional, Nayarit ha presentado una tasa de crecimiento del pIB por debajo de la media nacional, situación que lo ubica como uno de los estados de la República con menores niveles de crecimiento y, aunado a ello, un limitado nivel de desarrollo. ${ }^{10}$

Por otra parte, y también en una mirada retrospectiva, las estadísticas del PIB para Nayarit parecen mostrar una convergencia en la especialización económica de la entidad desde las actividades agropecuarias a las del sector terciario y, de manera más puntual, a los subsectores de comercio y servicios (cuadro 1), concentradas principalmente en la capital del estado y zonas turísticas del municipio de Bahía de Banderas. ${ }^{11}$

${ }^{9}$ El espacio que ocupa esta entidad representa $1.4 \%$ de la superficie total del país, por lo que es un estado relativamente pequeño $\left(27,815 \mathrm{~km}^{2}\right)$, se ubica en el lugar número 23 en relación con los demás estados de la República Mexicana. Bahía de Banderas es el municipio más joven de Nayarit. Mediante decreto expedido en diciembre de 1989 se oficializa como nuevo municipio, el número 20, separándolo territorialmente del municipio de Compostela.

${ }^{10}$ Según el informe del Consejo Nacional de Población (Conapo) de 2005, los tres municipios de la sierra nayarita (El Nayar, La Yesca y Huajicori) presentan muy alto grado de marginación, incluso, el primero de ellos se ubica en el nada halagüeño tercer lugar de todo el país (La Yesca en el 164 y Huajicori en el 269). Otros seis municipios se ubican en nivel medio de marginación (Conapo, 2006: 197-199).

${ }^{11}$ En los últimos 20 años, y de manera más acelerada los 10 recientes, la costa sur de Nayarit (sobre todo el municipio de Bahía de Banderas) se transformó de manera importante, la actividad turística ha tenido inversiones cuantiosas, en especial en infraestructura carretera y complejos turísticos. Económica y demográficamente es la región más dinámica del estado. La expansión de la actividad turística se ha dado en relación con el destino de Puerto Vallarta, asimismo, los vínculos laborales, comerciales, de uso del tiempo libre, de relaciones personales también tienen lugar en mayor medida con Puerto Vallarta, Jalisco, que con la población de la zona rural del mismo municipio y del de Compostela. Así, las actividades de las personas están supeditadas a un uso horario diferente 


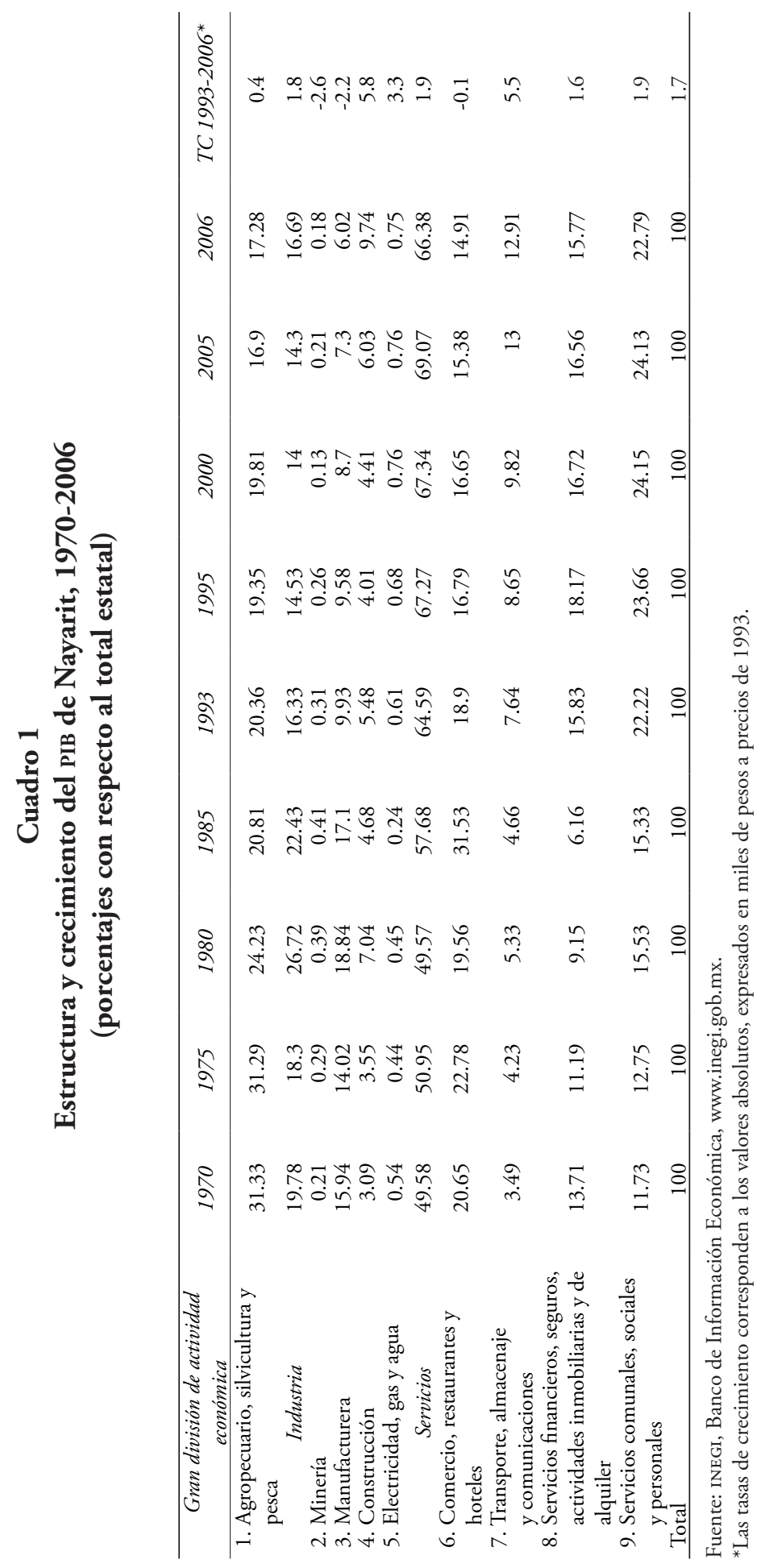


Prácticamente desde los ańos noventa, más de 60\% del pIB estatal lo conforma el sector terciario (comercio y servicios), dando lugar a lo que Garza $(2006,2008)$ llama un proceso de servicialización de la economía. A manera de ejemplo, en 2006 sobresalen en primer lugar los servicios comunales, sociales y personales (con una contribución de $22.79 \%$ al PIB estatal), mientras que ese mismo año el sector agropecuario aporta $17.28 \%$, superior al $16.69 \%$ del sector industrial en su conjunto. Así, aunado al auge del sector servicios en la economía del Nayarit contemporáneo, se encuentra un estancamiento de la industria, de hecho, tanto la industria manufacturera como la minería presentan tasas de crecimiento negativas (-2.2 y -2.6, respectivamente) durante el periodo 1993-2006 (cuadro 1).

No obstante, a pesar del deterioro en la aportación del sector agropecuario al PIB estatal, éste sigue desempeñando un papel central en el mosaico de actividades que integran la economía nayarita, además de que en buena parte la agricultura es el soporte de las principales actividades industriales y de comercio en la entidad. Se producen alrededor de 70 cultivos diferentes, entre los que destacan: tabaco, frijol, maíz y caña de azúcar, por lo que no es casualidad que el beneficio del tabaco, la molienda de azúcar y la producción manufacturada de harina de maíz constituyan los principales giros industriales en la entidad (Madera, 2006). Mencionamos que en 2006 el sector agropecuario contribuía con 17.28\% del PIB estatal, muy por encima del $6.02 \%$ que aporta la industria manufacturera y también superior al $14.91 \%$ de comercios, restaurantes y hoteles. También ese mismo año, el pIB de la industria manufacturera fue de 570,478 millones de pesos a precios de 1993; y de éste, 80\% corresponde a la rama de productos alimenticios, bebidas y tabaco. Asimismo, la estructura comercial de Nayarit es de carácter minorista y se concentra alrededor de las ciudades que, a excepción de Tepic y Bahía de Banderas, tienen una dinámica impulsada por las labores agrícolas que se desarrollan en sus alrededores. ${ }^{12}$

(Jalisco tiene un uso horario del centro, mientras que el de Nayarit se rige por el del Pacífico), sin embargo, la población ha sabido adaptarse a estos procesos (Madera y Garrafa, 2007).

${ }^{12}$ Acaponeta, por ejemplo, tradicionalmente ha sido una población donde el comercio en pequeña escala, junto con las actividades agrícolas, son las actividades que le da vida, y más aún en épocas de buena cosecha. Desde tiempos precortesianos Acaponeta se ha considerado lugar de afluencia de los pueblos de las regiones serranas de Durango y Nayarit, de la gente de los valles, de gran parte de la costa y de lo que hoy es el sur de Sinaloa, que se daban cita ahí para hacer sus trueques comerciales de sal, camarones y pescado seco de la costa, por miel, bules, carne y pieles de venado de la sierra, y otros muchos productos más de aquellos años (Chávez, 1983: 15). Todavía en la actualidad, Acaponeta es el lugar de cita de muchos pueblos indígenas y mestizos de las sierras cercanas, así como de poblaciones de los valles y zonas costeras del norte de Nayarit y el sur de Sinaloa. Es muchísima la gente que llega a Acaponeta a comprar y a vender, así como jóvenes que vienen a seguir sus estudios de los niveles de bachillerato y superior (Madera, 2006: 74). 
Vale destacar además que si se observan las aportaciones estatales a la economía nacional, la del sector agropecuario es superior al de la economía nayarita en su conjunto (cuadro 2), así como al de todos los sectores en lo individual, que por cierto, también muestran una tendencia a la baja, a excepción de los sectores de construcción y los servicios comunales, sociales y personales que tienen un ligero aumento: de 0.75 a $1.26 \%$ y de 0.64 a $0.69 \%$, respectivamente cada uno de ellos, durante el periodo 1993-2006 (cuadro 2).

Otro indicador que, a pesar de la servicialización de la economía en Nayarit, sigue resaltando la vigencia del sector agropecuario, es la proporción de población ocupada en él por municipios. Así, en el año 2000 la población ocupada en el sector primario representaba $27.8 \%$, proporción que es fácilmente rebasada por la mayoría de los municipios (por ejemplo, $62.7 \%$ en Huajicori, $60.4 \%$ en Rosamorada, $54.1 \%$ en San Blas y $53.5 \%$ en Santiago Ixcuintla), a excepción de Tepic, Bahía de Banderas e Ixtlán del Río, donde más de $60 \%$ de su población ocupada se ubica en el sector de servicios (cuadro 3).

Así, el crecimiento de las economías no se explica sólo por el incremento de su dotación de capital físico, sino también por el aumento del capital humano disponible como resultado de los avances en materia de educación y capacitación de la fuerza laboral (Ocegueda, 2003b: 39). De acuerdo con tales indicadores de capital humano, de 1980 a 2005 en el estado de Nayarit disminuyó tanto la población de 15 años y más con estudios de primaria, como la que no tiene grado alguno de escolaridad (en el mismo rango de edad). Por su parte, la población de 15 años y mayor con estudios de secundaria y más se ven incrementadas de manera significativa que, a su vez, permiten aumentar la escolaridad promedio de 6.3 a 8 ańos (cuadro 4).

Como bien señala Ocegueda (2003b: 39-40), tales incrementos resultan fundamentales, sobre todo por el rango de edad en cuestión, población económicamente activa, mismos que se asocian (o debieran asociarse, tal vez sería mejor dicho) con la adquisición de mayores conocimientos y habilidades y, por ende, con un mejor desempeño laboral. Habría que verlo.

\subsection{El sector servicios en Nayarit, 1980-2003}

Una vez revisada la estructura y comportamiento de la economía nayarita en su conjunto, en este apartado nos interesa mostrar un repaso breve de la dinámica y estructura de las actividades terciarias en el estado de Nayarit, tomando como base la información de los VIII, X, XI, XII y XIII censos de comercio y servicios del INEGI, mediante una compleja y mi- 


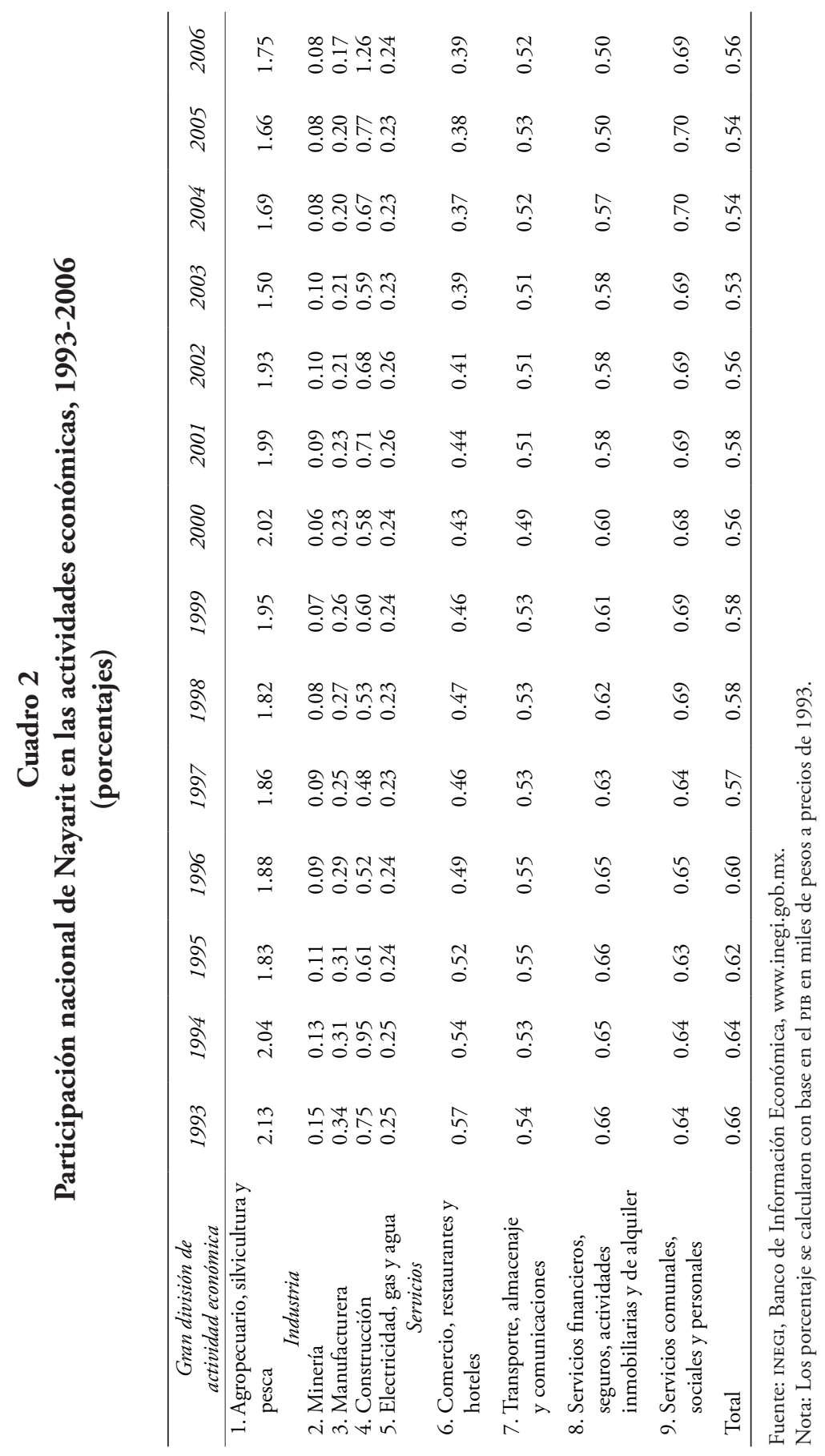




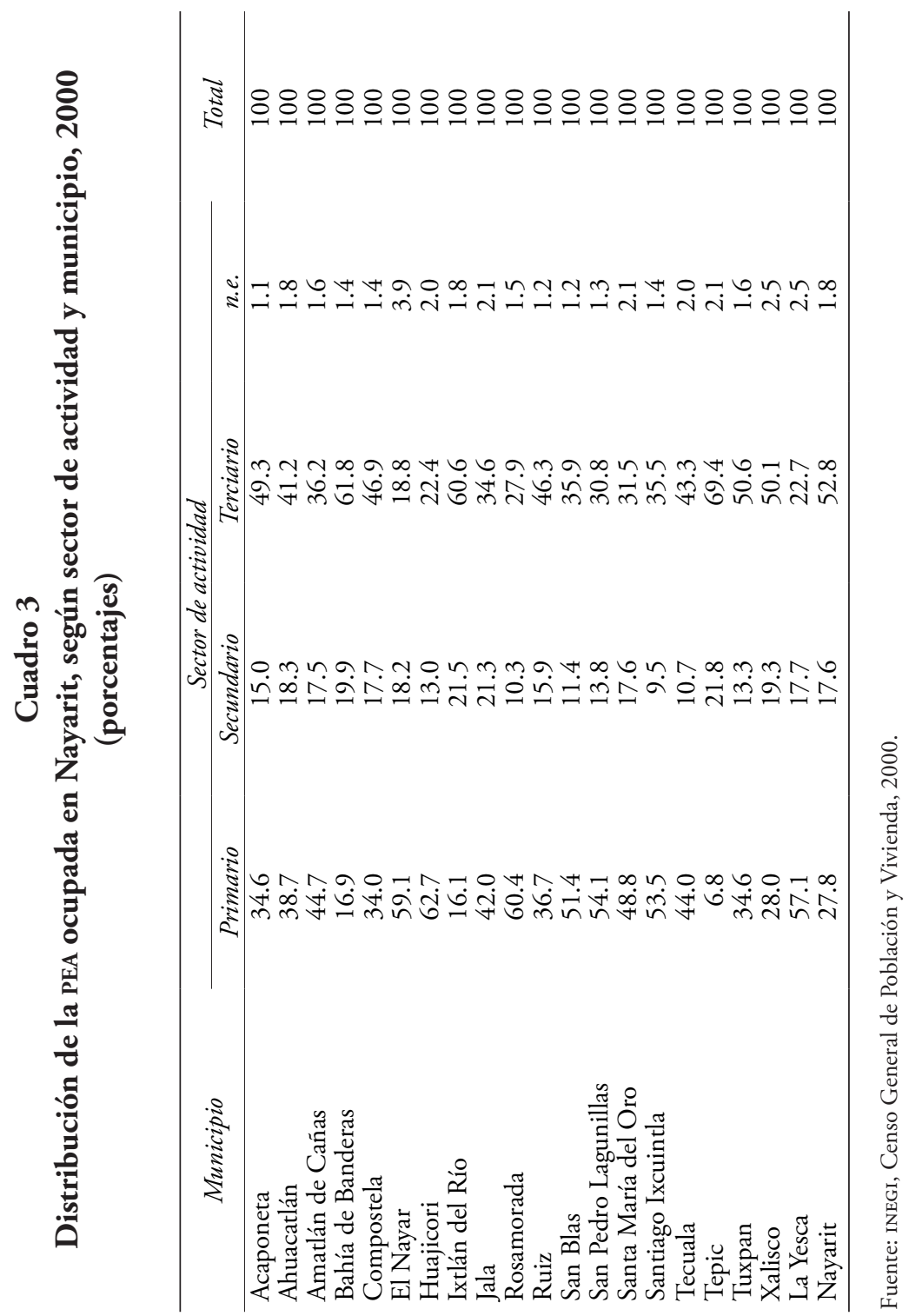




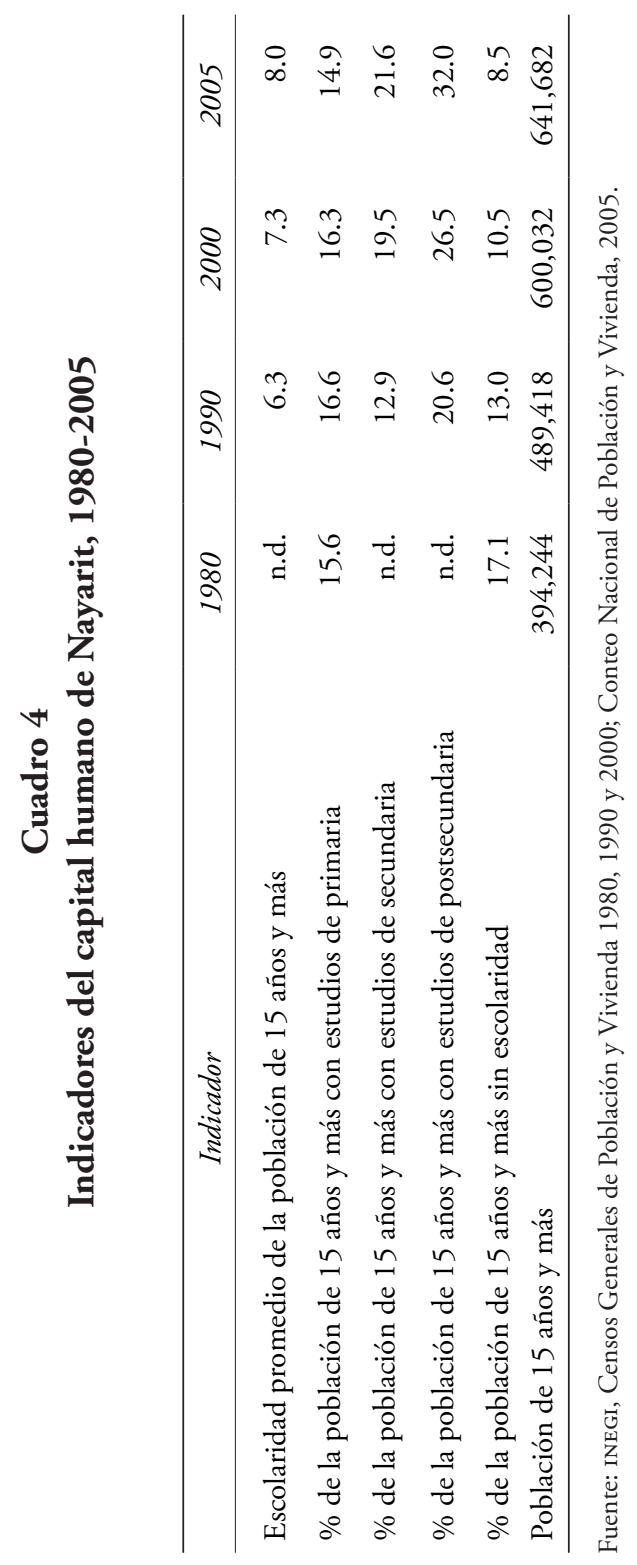


nuciosa metodología desarrollada por Garza (2008) en los apéndices metodológicos 1 y 2 .

Durante el periodo analizado se pueden destacar dos etapas en el comportamiento de la aportación de Nayarit, mediante las 17 ramas terciarias consideradas en la metodología, al рів nacional (cuadro 5). La primera de ellas (1980-1988) muestra un claro repunte del sector terciario, que pasó de $0.4 \%$ en 1980 a un nada despreciable $0.7 \%$ en 1988 , año en que se presenta un quiebre para dar paso a la segunda etapa (19882003), donde se reduce dicha participación; otra vez llegó a apenas $0.4 \%$ en el año 2003.

Este quiebre encuentra parte de su explicación en los cambios que se comienzan a vivir, desde principios de la década de 1980, en la política de la economía mexicana y que, de manera especial, impactan en la economía nayarita con la desaparición de Tabamex en el año de 1989 (recordemos que la producción y agroindustria del tabaco es una de las actividades clave en la economía nayarita, de hecho, alrededor de $80 \%$ tanto de la superficie cultivada como del número de productores en México, se encuentra en la zona de Nayarit), aunque es un proceso que comienza a principios de los ochenta con el gobierno de Miguel de la Madrid, a raíz de la política de modernización del Estado mexicano que implicó la desaparición de la mayor parte de las empresas de participación estatal.

En el grupo terciario (II) se concentra la mayor participación a la economía nacional, de comercio y servicios al consumidor, que en todo momento es superior a la aportación media de las 17 ramas en su conjunto, siguiendo además una tendencia similar a dicha media (cuadro 5). En el grupo II sobresalen el comercio de alimentos, bebidas y tabaco, así como el de gasolinerías y combustibles.

Las ramas que se ven beneficiadas con el quiebre de la participación del sector terciario al PIB nacional, son las tiendas departamentales, por una parte, y por la otra la de hoteles, moteles y posadas (cuadro 5), que en los años de recesión tienen una participación muy superior a la media estatal.

\section{Especialización y productividad en Nayarit, la metodología utilizada}

\subsection{Indice de especialización}

Conocer la estructura económica de una región es un punto de referencia importante. Para ello planteamos la utilización del índice de especializa- 


\section{Cuadro 5}

Nayarit: PIB por grupos de actividad del sector servicios, 1980-2003 (porcentajes respecto al total nacional)

\begin{tabular}{|c|c|c|c|c|c|}
\hline Grupo $/$ denominación & 1980 & 1988 & 1993 & 1998 & 2003 \\
\hline I. Comercio y servicios al productor & 0.2 & 0.4 & 0.2 & 0.4 & 0.3 \\
\hline 1. Servicios profesionales & 0.1 & 0.3 & 0.2 & 0.4 & 0.3 \\
\hline 721 servicios profesionales a empresas & 0.1 & 0.3 & 0.2 & 0.4 & 0.3 \\
\hline 2. Comercio de bienes de capital e intermedios & 0.3 & 0.5 & 0.2 & 0.4 & 0.4 \\
\hline 731 comercios al mayoreo & 0.3 & 0.7 & 0.6 & 0.6 & 0.6 \\
\hline $\begin{array}{l}732 \text { comercios de equipo e insumos } \\
\text { industriales }\end{array}$ & 0.3 & 0.3 & 0.0 & 0.2 & 0.3 \\
\hline II. Comercio y servicios al consumidor & 0.6 & 0.9 & 0.6 & 0.7 & 0.6 \\
\hline 3. Comercio de bienes de consumo inmediato & 0.8 & 1.0 & 0.8 & 0.8 & 0.7 \\
\hline $\begin{array}{l}811 \text { comercios de alimentos, bebidas } \\
\text { y tabaco }\end{array}$ & 1.2 & 1.2 & 1.0 & 0.8 & 0.8 \\
\hline 812 supermercados & 0.1 & 0.7 & 0.3 & 0.7 & 0.5 \\
\hline 813 gasolinerías y combustibles & 1.2 & 1.1 & 0.8 & 1.3 & 0.7 \\
\hline 4. Servicios de consumo inmediato & 0.5 & 1.0 & 0.5 & 1.0 & 0.8 \\
\hline 821 preparación de alimentos y bebidas & 0.9 & 1.3 & 0.7 & 0.7 & 0.7 \\
\hline 822 aseo y limpieza & 0.4 & 0.7 & 0.5 & 0.5 & 0.7 \\
\hline 823 recreación y esparcimiento & 0.2 & 0.5 & 0.7 & 0.4 & 1.0 \\
\hline 824 difusión e información & 0.4 & 0.4 & 0.2 & 0.7 & 0.2 \\
\hline 825 hoteles, moteles y posadas & 0.4 & 1.2 & 0.6 & 2.1 & 1.5 \\
\hline 5. Comercio de bienes de consumo duradero & 0.5 & 0.8 & 0.7 & 0.6 & 0.6 \\
\hline 831 bienes del hogar y personales & 0.5 & 0.9 & 0.6 & 0.6 & 0.6 \\
\hline 832 tiendas de departamento & 0.2 & 0.5 & 1.4 & 0.9 & 1.0 \\
\hline 833 automotrices y autopartes & 0.7 & 0.7 & 0.6 & 0.5 & 0.5 \\
\hline 6. Servicios de consumo duradero & 0.4 & 0.7 & 0.4 & 0.4 & 0.1 \\
\hline 841 reparaciones & 0.5 & 1.0 & 0.7 & 0.6 & 0.7 \\
\hline 842 educación y cultura privados & 0.2 & 0.4 & 0.3 & 0.2 & 0.3 \\
\hline 843 salud y asistencia social privada & 0.5 & 0.8 & 0.5 & 0.4 & 0.3 \\
\hline Total & 0.4 & 0.7 & 0.5 & 0.5 & 0.4 \\
\hline
\end{tabular}

Fuente: Cálculos realizados con base en información de los viII, X, XI, XII y XIII censos comerciales y de servicios, INEGI.

La información fue sujeta a un minucioso ejercicio de comparación intercensal y se ajustó con cuentas nacionales, se explica en los apéndices estadísticos 1 y 2 de Garza (2008).

${ }^{a}$ Las claves no corresponden con las que se utilizan en los censos, por la dificultad para uniformarlas pues se modifican en cada censo. Las que se presentan se seleccionaron únicamente para propósitos de esta investigación. 
ción normalizado propuesto por Dalum et al. (1999),,$^{13}$ para calcular usando el personal ocupado en cada uno de los subgrupos de actividad planteados por Garza (2008), en los sectores comercio y servicios. Los datos utilizados para el cálculo del índice se obtuvieron de los censos comercial y de servicios de 1981, 1989, 1994, 1999 y 2004 del INEGI.

La versión normalizada del índice propuesta por Dalum et al (1999) se expresa como:

$$
I E_{i}^{*}=\left\{\left[\left(L_{i E} / L_{S E}\right) /\left(L_{i N} / L_{S N}\right)\right]-1\right\} /\left\{\left[\left(L_{i E} / L_{S E}\right) /\left(L_{i N} / L_{S N}\right)\right]+1\right\}
$$

Donde los subíndices $i$ y $s$ se refieren al grupo y al sector, mientras que $E$ y $N$ aluden al carácter estatal y nacional de la información, respectivamente.

De acuerdo con Ocegueda (2005), los valores de la especialización negativa van de $[-1,0)$ y para la especialización positiva se sitúan entre $(0,1]$, y mediante el signo del índice se pueden distinguir los procesos de especialización y desespecialización: si es positivo, la economía se especializa y, si es negativo, sucede lo contrario, mientras que cuando el valor es igual a cero se habla de una especialización neutral.

El beneficio que presenta este indicador es que permite identificar si las actividades al interior del estado están tomando una posición relevante respecto a lo que ocurre en el ámbito nacional, aunque cabe señalar que obtener valores positivos no siempre se relaciona con una actividad más dinámica o, en caso contrario, con un estancamiento. Lo anterior se puede interpretar en el sentido de que es factible encontrar procesos de especialización basados en un decrecimiento más lento de cierta actividad económica en el estado con respecto a lo que ocurre en el país y viceversa (Ocegueda, 2006).

\subsection{El coeficiente de Verdoorn}

Para determinar el coeficiente de productividad (Verdoorn), y por medio de él establecer el tipo de rendimientos que presenta cada grupo de actividad, se utilizó el planteamiento de León (1998), derivado de una función de producción de tipo $Q=A_{o} e^{\pi t} F^{w}$, tomando logaritmos y diferenciando con respecto al tiempo se obtiene,

$$
q=\pi+w F
$$

\footnotetext{
${ }^{13}$ Tomando en cuenta las ventajas que presenta con respecto al índice de especialización calculado como $I E_{i}=\left(L_{i E} / L_{S E}\right) /\left(L_{i N} / L_{S N}\right)$ en el sentido que una desespecialización puede tomar valores en el intervalo $[0,1)$, mientras que para una especialización positiva los valores se localizan en el intervalo $(1, \infty]$, así que el valor de 1 puede ser una especialización neutral, porque el cociente de la región y el del estado son iguales.
} 
Asumiendo que $\pi$ es la tasa de crecimiento autónoma y $w=(\alpha+\beta)$ representa el grado de rendimientos a escala de $F=K^{\delta} L^{1-\delta}$, y sustituyendo en la ecuación anterior resulta,

$$
q=\pi+(\alpha+\beta) F
$$

Presentado de manera endógena los factores de la producción, la ecuación se transforma en:

$$
F=-\frac{\pi}{\alpha+\beta}+q \frac{1}{\alpha+\beta}
$$

que al expresar en contribuciones del capital y el trabajo a la producción $\delta$ y $(1-\delta)$, respectivamente, resulta,

$$
\delta k+(1-\delta) l=-\frac{\pi}{\alpha+\beta}+q \frac{1}{\alpha+\beta}
$$

Suponiendo que $\phi=-\pi /(\alpha+\beta)$ y $\sigma=1 /(\alpha+\beta)$, presentando una forma simplificada como:

$$
\delta k+(1-\delta) l=\phi+\sigma q
$$

De la ecuación anterior se deriva el tipo de rendimientos en la actividad, es decir, serán crecientes a escala si $\sigma<1$, decrecientes si $\sigma>1$ y constantes cuando $\sigma=1$ debido a que $\alpha+\beta=1 / \sigma$. Para la estimación se utilizaron como proxies del empleo y del capital, sueldos y salarios y capital fijo, respectivamente, en tanto que como variable proxy del producto se utilizó el РІв.

\subsection{Método de estimación}

Con la información obtenida de los censos de comercio y servicios fue posible determinar un panel de datos, en el que se incluyen cuatro periodos (1980-1988, 1988-1993, 1993-1998 y 1998-2003), en tanto que las secciones cruzadas se determinaron por los grupos de actividad de los sectores establecidos por Garza (2008) a una escala de tres dígitos.

Para calcular el coeficiente de Verdoorn se utilizaron mínimos cuadrados ordinarios con $P C S E,{ }^{14}$ es decir, con corrección de error estándar de los estimadores asociados a la presencia de heterocedasticidad. La corrección

\footnotetext{
${ }^{14}$ Panel corrected standard error, planteado por Beck y Katz (1995), en el cual los residuales se reemplazan por el estimador de momentos para varianzas incondicionales, y cuyos resultados son consistentes al no restringir la varianza incondicional $\Omega_{M}$, sin embargo, establece restricciones adicionales a la matriz de covarianza condicional.
} 
se utilizó para sección cruzada, en la que se reemplaza el producto de los residuales utilizando el método de White, por un estimado de los residuales de sección cruzada de la matriz de covarianza, el cual se expresa como:

$$
\left(\frac{N^{*}}{N^{*}-K^{*}}\right)\left(\sum_{t} X_{t}^{\prime} X_{t}\right)^{-1}\left(\sum_{t} X_{t}^{\prime} \hat{\mathbf{\Omega}}_{M} X_{t}\right)\left(\sum_{t} X_{t}^{\prime} X_{t}\right)^{-1}
$$

La expresión anterior presenta la corrección propuesta por White (1980) y Beck y Katz (1995) en la matriz de varianza y covarianza tradicional, es decir, $\Omega_{M}$ es la matriz de varianza incondicional que es ponderada por el número de secciones cruzadas y el número total de parámetros estimados, $N^{*}$ y $K^{*}$, respectivamente. Aun cuando los resultados de este estimador son robustos respecto a la heterocedasticidad de secciones cruzadas, no lo son con respecto a la correlación general entre los residuales y sólo utiliza los elementos de la diagonal $\Omega_{M}$.

Tomando como base la ecuación para calcular el coeficiente de Verdoorn derivada en la sección anterior, y transformarla en la especificación para panel de datos, se obtienen dos ecuaciones alternativas:

$$
\begin{aligned}
& (\delta k+(1-\delta) l) Y_{i t}=\phi+\sigma X_{i t}+u_{i t} \\
& (\delta k+(1-\delta) l) Y_{i t}=\phi+\sigma_{i} X_{i t}+u_{i t}
\end{aligned}
$$

La diferencia entre ambas es el subíndice $i$ de $\sigma$ de la segunda ecuación que indica el cálculo de pendientes diferentes para cada sección cruzada. Con el objetivo de determinar cuál de estas especificaciones es la más adecuada se plantearon las siguientes pruebas de hipótesis, las cuales podrán establecer si los grupos de actividad tienen la misma pendiente o es diferente en cada uno de ellos. Siguiendo el planteamiento de Pindyck y Rubinfeld (1998), el estadístico utilizado se plantea como sigue:

$$
F_{N+T-2, N T-N-T}=\frac{\left(E S S_{1}-E_{2}\right) /(\mathrm{N}+\mathrm{T}-2)}{E S S_{2} /(N T-N-T)}
$$

En el cual $N$ y $T$ representan el número de secciones cruzadas y el número de observaciones, respectivamente, en tanto que $E S S_{1}$ y $E S S_{2}$ representan la suma de los cuadrados del error en cada una de las especificaciones de panel, respectivamente. El resultado del estadístico estimado se contrastó con la distribución $F$ al nivel de significancia de 5\%, en el cual las hipótesis de prueba fueron: 
$H_{0}$ : pendientes iguales entre ramas.

$H_{1}$ : pendientes diferentes entre ramas.

\section{Especialización y productividad en Nayarit, el análisis empírico}

\subsection{Indice de especialización}

El índice de especialización del empleo para el periodo 1980-2003 (cuadro 6), arroja que en el caso de los grupos de actividad relacionados con comercio y servicios al productor sólo existe especialización positiva en el comercio al mayoreo (731), especialización que ha aumentado con el paso del tiempo, ya que en 1988 el índice arrojaba un valor de 0.057 , en tanto que para el año 2003, este valor ascendió a 0.138 , señalando así el aumento personal en esta actividad.

En cuanto a las actividades relacionadas con el comercio y los servicios al consumidor, se observa que de manera agregada el grupo de actividad enfocado al comercio de bienes de consumo inmediato presenta una especialización positiva en todo el periodo, aunque con una ligera disminución al final del mismo, ya que de 0.039 en 1980, pasó a 0.029 en 2003. En este grupo, la actividad que presenta una especialización positiva mayor es la relacionada con el comercio de bebidas y tabaco (811).

Respecto al grupo dedicado a los servicios de consumo inmediato, éste presenta una especialización positiva más fuerte, misma que se duplicó entre el inicio y el final del periodo; ya que de presentar un índice de 0.115 en 1980, para 2003 aumentó a 0.237. En este grupo la actividad que presenta un mayor efecto es la que se relaciona con hoteles, moteles y posadas (825), mismo que se ha incrementado de manera significativa entre 1988 y 2003: de 0.184 pasó a 0.593, respectivamente. Lo anterior podemos asociarlo al impulso que comenzó a darse en la costa sur del estado, primero con la creación del municipio de Bahía de Banderas (1989) y, recientemente, con la Riviera Nayarit.

El comercio de bienes de consumo duradero sólo presenta especialización positiva en la última parte del periodo (1998 y 2003), principalmente asociados al comercio de bienes de productos del hogar y personales (831), cuyos índices fueron de 0.046 y 0.060 , respectivamente.

El último grupo de actividad, dedicado a los servicios de consumo duradero, presenta especialización positiva en todo el periodo únicamente en la actividad relacionada con las reparaciones (841), en tanto que los servicios de asistencia social y privada (843) lo hacen sólo en los años 1988 y 1998. En relación con los servicios educativos de carácter privado (842), aun cuando no presenta especialización positiva, se nota un incremento, ya que al principio del periodo el valor del índice era de -0.525 y 
al final del mismo el valor fue de -0.167 , mismo que podemos relacionar con el aumento reciente de escuelas privadas en todo el estado.

\subsection{Estimación del coeficiente de Verdoorn}

Mediante la estimación del coeficiente de Verdoorn para el sector de servicios en su conjunto, los resultados arrojan que un aumento de $10 \%$ en la producción se relaciona con un incremento en la productividad del empleo de $7.6 \%$, que es consistente con los resultados teóricamente esperados, lo que indica la existencia de rendimientos crecientes a escala del orden de $31 \%$ (cuadro 7 ).

De manera alternativa se estimó el coeficiente de Verdoorn para el sector mediante la especificación de interceptos diferentes, y se encontró que el grupo de tiendas de departamento (832) presenta una relación con la productividad cercana a $1.5 \%$ ante un aumento de la producción de $10 \%$, con rendimientos crecientes a escala de 600 por ciento.

Se encontraron rendimientos crecientes a escala en los siguientes grupos de actividad: servicios profesionales a empresas (721), gasolineras y combustibles (813) y reparaciones (841); así como en el comercio de alimentos, bebidas y tabaco (811), aseo y limpieza (822), y en salud y asistencia social y privada (843), aunque con menores niveles de probabilidad del orden del 75 y $70 \%$, respectivamente. En el resto de las ramas no se obtuvo evidencia del tipo de rendimientos que presenta, debido a la insignificancia estadística que presentan (cuadro 8).

En función de los resultados obtenidos en las ecuaciones de regresión anteriores, se realizó una prueba de hipótesis con el propósito de determinar cuál es la correcta especificación encontrándose que al 95\% de probabilidad la correcta especificación del sector es la correspondiente a interceptos iguales, donde se evidencia la existencia de rendimientos crecientes a escala del orden del 31\% (Cuadro 9).

\section{Consideraciones finales}

El crecimiento económico ha sido tema de discusión constante, sin embargo, en el ámbito de las regiones el estudio ha aumentado en tiempos recientes, resaltando la importancia de lo local y la responsabilidad de los actores y las instituciones en el crecimiento y desarrollo regional.

En el análisis de las teorías del crecimiento económico destaca el planteamiento kaldoriano, que constituye una herramienta importante en la economía, pues permite identificar los sectores y actividades que presentan rendimientos crecientes a escala tanto de carácter dinámico como estático. Mediante el cálculo de la ley de Verdoorn, se encontró que en Nayarit el 


\section{Cuadro 6}

Nayarit: índice de especialización normalizado por grupos de actividad en el sector servicios, 1980-2003 (personal ocupado)

\begin{tabular}{|c|c|c|c|c|c|}
\hline Grupo /denominación & 1980 & 1988 & 1993 & 1998 & 2003 \\
\hline I. Comercio y servicios al productor & -0.210 & -0.115 & -0.304 & -0.145 & -0.082 \\
\hline 1. Servicios profesionales & -0.242 & -0.083 & -0.209 & -0.191 & -0.127 \\
\hline $\begin{array}{l}721 \text { servicios profesionales a } \\
\text { empresas }\end{array}$ & -0.242 & -0.083 & -0.209 & -0.191 & -0.127 \\
\hline $\begin{array}{l}\text { 2. Comercio de bienes de capital e } \\
\text { intermedios }\end{array}$ & -0.192 & -0.153 & -0.450 & -0.074 & -0.005 \\
\hline 731 comercios al mayoreo & -0.164 & 0.057 & -0.024 & 0.102 & 0.138 \\
\hline $\begin{array}{l}732 \text { comercios de equipo e } \\
\text { insumos industriales }\end{array}$ & -0.219 & -0.326 & -0.987 & -0.224 & -0.143 \\
\hline II. Comercio y servicios al consumidor & 0.039 & 0.031 & 0.019 & 0.053 & 0.029 \\
\hline $\begin{array}{l}\text { 3. Comercio de bienes de consumo } \\
\text { inmediato }\end{array}$ & 0.137 & 0.041 & 0.077 & 0.058 & 0.074 \\
\hline $\begin{array}{l}811 \text { comercios de alimentos, } \\
\text { bebidas y tabaco }\end{array}$ & 0.182 & 0.076 & 0.109 & 0.093 & 0.100 \\
\hline 812 Supermercados & -0.394 & -0.163 & -0.190 & -0.111 & -0.039 \\
\hline combustibles & 0.042 & 0.029 & 0.126 & -0.008 & 0.079 \\
\hline 4. Servicios de consumo inmediato & 0.115 & 0.141 & 0.059 & 0.127 & 0.237 \\
\hline $\begin{array}{l}821 \text { preparación de alimentos y } \\
\text { bebidas }\end{array}$ & 0.206 & 0.160 & 0.074 & 0.113 & 0.107 \\
\hline 822 aseo y limpieza & -0.101 & -0.098 & -0.128 & -0.048 & 0.073 \\
\hline parcimiento & -0.086 & 0.152 & 0.071 & -0.002 & 0.261 \\
\hline 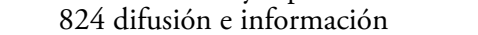 & & & & 03 & -0.278 \\
\hline 825 hoteles, moteles y posadas & -0.156 & 0.184 & 0.181 & 0.304 & 0.593 \\
\hline $\begin{array}{l}\text { 5. Comercio de bienes de consumo } \\
\text { duradero }\end{array}$ & -0.091 & -0.068 & -0.050 & 0.029 & 0.037 \\
\hline 831 bienes del hogar y personales & -0.048 & -0.061 & -0.041 & 0.046 & 0.060 \\
\hline & & & & -0.176 & -0.214 \\
\hline 833 au & -0.089 & -0.050 & -0.029 & -0.015 & -0.018 \\
\hline 6. Servicios de consumo duradero & -0.127 & 0.001 & -0.041 & -0.015 & -0.646 \\
\hline 841 reparaciones & 0.008 & 0.052 & 0.062 & 0.082 & 0.106 \\
\hline 842 educación y cultura privados & -0.525 & -0.253 & -0.182 & -0.229 & -0.167 \\
\hline $\begin{array}{l}843 \text { salud y asistencia social } \\
\text { privada }\end{array}$ & -0.008 & 0.116 & -0.099 & 0.022 & -0.048 \\
\hline
\end{tabular}

Fuente y llamada ${ }^{a}$ : igual que el cuadro 5.

\section{Cuadro 7}

Nayarit: estimación del coeficiente de Verdoorn para el sector servicios, 1980-2003 (interceptos comunes)

\begin{tabular}{|c|c|c|c|c|}
\hline & & & & Rendimientos \\
\hline \multirow{3}{*}{$\mathrm{C}$} & $\phi$ & $\sigma$ & $T$ & $1 / \sigma$ \\
\hline & 0.0749 & & 3.8895 & \\
\hline & & 0.763104 & 12.4304 & $\begin{array}{r}1.3104 \\
\end{array}$ \\
\hline $\begin{array}{r}\mathrm{R}^{2}= \\
\mathrm{N}^{*}=\end{array}$ & $\begin{array}{r}0.6976 \\
17\end{array}$ & & $\mathrm{~F}=$ & 191.4787 \\
\hline
\end{tabular}

Fuente: Igual que el cuadro 5.

${ }^{*} \mathrm{~N}$ se refiere al número de secciones cruzadas por cada uno de los periodos utilizados: 1980-1988, 1988-1993, 1993-1998 y 1998-2003. 


\section{Cuadro 8 \\ Nayarit: estimación del coeficiente de Verdoorn para el sector servicios, 1980-2003}

\begin{tabular}{|c|c|c|c|c|}
\hline & & & & Rendimientos \\
\hline & $\Phi$ & $\sigma$ & $T$ & $1 / \sigma$ \\
\hline C & 0.7814 & & 10.9913 & \\
\hline$S 721^{\mathrm{b}}$ & & 0.0908 & 1.0208 & 11.0173 \\
\hline S731 & & 0.0189 & 0.2126 & N.S. \\
\hline S732 & & -0.0053 & -0.0601 & N.S. \\
\hline S81 $1^{\mathrm{c}}$ & & 0.1037 & 1.1662 & 9.6439 \\
\hline S812 & & 0.0524 & 0.5924 & N.S. \\
\hline $\mathrm{S} 813^{\mathrm{b}}$ & & 0.0957 & 1.0786 & 10.4486 \\
\hline S821 & & 0.0741 & 0.8336 & N.S. \\
\hline S822 & & 0.1056 & 1.1863 & 9.4711 \\
\hline S823 & & 0.0764 & 0.8535 & N.S. \\
\hline S824 & & 0.0531 & 0.5975 & N.S. \\
\hline S825 & & 0.0500 & 0.5631 & N.S. \\
\hline S831 & & 0.0566 & 0.6370 & N.S. \\
\hline$S 832^{a}$ & & 0.1519 & 1.7000 & 6.5824 \\
\hline S833 & & 0.0636 & 0.7149 & N.S. \\
\hline S841 ${ }^{\mathrm{b}}$ & & 0.0903 & 1.0153 & 11.0798 \\
\hline S842 & & 0.0616 & 0.6904 & N.S. \\
\hline S843c & & 0.1128 & 1.2682 & 8.8687 \\
\hline $\mathrm{R} 2=$ & 0.7147 & & $\mathrm{~F}=$ & 7.36898 \\
\hline $\mathrm{N}^{*}=$ & 17 & & & \\
\hline
\end{tabular}

Fuente: Igual que el cuadro 7.

${ }^{*} \mathrm{~N}$, véase el cuadro 7 .

a,b,c: Indica que es estadísticamente significativo en 95,75 y $70 \%$ de probabilidad, respectivamente.

\section{Cuadro 9}

Nayarit: prueba de especificación F, sector servicios, 1980-2003

\begin{tabular}{cccccccccc}
\hline & ESS1 & ESS2 & $N$ & $T$ & FCALC & GDLN & GDLD & FTAB & $H_{0}$ \\
\hline Nayarit & 1.6660 & 1.5809 & 17 & 4 & 0.1332 & 23 & 13 & 2.24 & Se acepta \\
\hline
\end{tabular}

Fuente: Cálculos realizados con base en resultados de los cuadros 7 y 8 .

sector servicios opera con rendimientos crecientes a escala de nivel agregado. En cuanto a los grupos de actividad la evidencia estadística es más débil, sin embargo, nos muestra que las actividades que operan con rendimientos crecientes a escala son sobre todo tiendas departamentales (832), seguido del grupo relacionado con servicios profesionales a empresas y reparaciones (721 y 841, respectivamente), actividades que destacan por la necesidad de cierto grado de especialización y cualificación, evidenciando la importancia de la formación de capital humano como motor de crecimiento. Finalmente, de manera más débil aparecen comercio de ali- 
mentos, bebidas y tabaco (811), servicios de aseo y limpieza (812), así como servicios de salud y asistencia social y privada (843).

En torno a la especialización, cabe señalar que en todos los grupos de actividad identificados, los índices estimados encuentran un aumento en la especialización positiva, o bien una reducción en la desespecialización, con respecto a lo que ocurre en el ámbito nacional. Sin embargo, lo paradójico de estos resultados es que la especialización positiva se presenta principalmente en actividades en las que no se encontró evidencia de rendimientos crecientes a escala, evidenciando con esto que la especialización económica que caracteriza a Nayarit es de carácter smithiano, es decir, la economía se especializa en actividades sin potencial para ser motores de crecimiento, al menos en el sector servicios.

De esta manera, es evidente la importancia estratégica de una política de gobierno capaz de incidir tanto en las mejoras de la calidad de vida de los nayaritas, como en el impulso al desarrollo económico local. Es necesaria una mirada retrospectiva a las políticas de desarrollo económico aplicadas hasta hoy en Nayarit, sus pretensiones y sus efectos, al mismo tiempo, es importante insistir en el reconocimiento y potencialización de las particularidades propias de cada una de las zonas económicas de Nayarit. Para ello, debe partirse, en primera instancia, de la concientización en los gobiernos locales, de la necesidad de políticas de este tipo, donde tales gobiernos participen de manera activa en el desarrollo económico local.

\section{Bibliografía}

Bairam, Erkin (1987), "The Verdoorn Law, returns to scale and industrial growth: a review of the literature", Australian Economic Papers, 26 (48), Blackwell Publishing, Finders, Australia, pp. 20-42.

Barrón, Karla (2009), Especialización y crecimiento económico en el estado de Nayarit: un enfoque postkeynesiano, Universidad Autónoma de Nayarit, México.

Beck, Nathaniel y Jonathan Katz (1995), "What to do (and not to do) with Time-Series-Cross-Section Data", The American Political Science Review, 89 (3), American Political Science Association, Los Ángeles, pp. 634-647.

Chacholiades, Miltiades (1992), Economía internacional, McGraw Hill, México. 
Chávez Gradilla, Néstor (1983), Breve bosquejo histórico descriptivo de la ciudad de Acaponeta y de los pueblos y lugares circunvecinos del norte del estado de Nayarit, Costa Amic, México.

Cimoli, Mario, Macio Holland, Gabriel Porcille, Annalise Primi y Sebastian Vergara (2006), "Growth, structural change and technological capabilities. Latin America in a comparative perspective", LEM Paper Series, 11, Sant'Anna School of Advanced Studies, Pisa, pp. 1-33.

Conapo (Consejo Nacional de Población (2006), Índices de marginación 2005, Conapo, México.

Dalum, Bent, Keld Laursen y Bart Verspagen (1999), "Does specialization matter for growth?", Industrial and Corporate Change, 8 (2), Oxford University Press, Oxford, pp. 267-288.

Dixon, Robert y Anthony Thirlwall (1975), "A model of regional growthrate differences on Kaldorian Lines", Oxford Economic Papers, 27 (2), Oxford University Press, Oxford, pp. 201-214.

Ezcurra, Roberto, Pedro Pascual y Manuel Rapún (2002), Disparidades espaciales en productividad y estructura sectorial de las regiones europeas, Universidad Pública de Navarra, Navarra.

Garza, Gustavo (2005), La urbanización de México en el siglo XX, El Colegio de México, México.

Garza, Gustavo (coord.) (2006), La organización espacial del sector servicios en México, El Colegio de México, México.

Garza, Gustavo (2008), Macroeconomía del sector servicios en la ciudad de México, 1960-2003, El Colegio de México México.

Grossman, Gene y Elhanan Helpman (1991), Innovation and growth in the global economy, MIT Press, Massachusetts.

Jungmittag, Andre (2004), "Innovations, technological specialization and economic growth in the USA", International Economics and Economic Policy, 1 (2), Heilderberg, pp. 247-273. 
Kaldor, Nicholas (1966), Causes of the slow rate of economic growth in the United Kingdom, Cambridge University Press, Cambridge.

Kaldor, Nicholas (1970), “The case for regional policies", Scottish Journal of Political Economy, 17 (3), Scottish Economic Society, Stirling, pp. 337-348.

Katz, Jorge (1968), "'Verdoorn effects', returns to scale, and the elasticity of factor substitution", Oxford Economic Papers, 20 (3), Oxford University Press, Oxford, pp. 342-352.

Klenow, Peter y Andrés Rodríguez-Clare (2004), "Externalities and growth", nBer Working Paper, Cambridge, MA.

Laursen, Keld (1998), "How structural change differs, and why it matters (for economic growth)", Danish Research Unit for Industrial Dynamics, Working Paper No. 98-25, DRUID, Copenhagen Business School, Copenhague.

León-Ledesma, Miguel Ángel (1998), "Verdoorn’s Law in the Spanish Regions", Working Paper, Keynes College, University of Kent.

León-Ledesma, Miguel Ángel y Anthony Thirlwall (2002), "The endogeneity of the natural rate of growth", Cambridge Journal of Economics, 26, Cambridge Political Economy Society, Cambridge, pp. 441-459.

Lucas, Robert (1988), "On the mechanics of economic development", Journal of Monetary Economics, 22 (1), Elsevier, Amsterdam, pp. $3-42$.

Madera Pacheco, Jesús (2006), "Las memorias y los silencios en la redefinición de lo campesino. La configuración de un modelo alternativo de desarrollo en la región tabacalera de Nayarit, México", tesis de doctorado, ISEC, Córdoba, España.

Madera Pacheco, Jesús y Olivia María Garrafa (2007), "Regiones agrícolas en Nayarit: cómo pensarlas a partir de los sujetos que las recrean", ponencia presentada en el vi Congreso de la AMER: La encrucijada del México rural. Contrastes regionales en un mundo desigual, Veracruz, México, 22-26 de octubre. 
McCombie John y John de Ridder (1983), "Kaldor's growth laws: Increasing returns, productivity, and output growth, the case of the United States", Journal of Post Keynesian Economics, 5 (3), ME Shape Inc y Armonk, Nueva York, pp. 373-388.

McCombie John y John de Ridder (1984), "The Verdoorn Law Controversy: some new empirical evidence using U.S. state data", Oxford Economic Papers, 36, Oxford, pp. 268-284.

McCombie, John, Maurizio Pugno y Bruno Soro (2002), "Introduction", en John McCombie, Maurizio Pugno y Bruno Soro (eds.), Productivity growth and economic performance. Essays on honor of P.J. Verdoorn, Palgrave McMillan, Hampshire, pp. 1-27.

Meliciani, Valentina (2001), Technology, trade and growth in OCDE countries: does specialization matter?, Routledge, Londres.

Ocegueda Hernández, Juan Manuel (2003a), "Análisis Kaldoriano del crecimiento económico en México", Comercio Exterior, 53 (11), México, pp. 1024-1034.

Ocegueda Hernández, Juan Manuel (2003b), "Estructura económica y especialización en Baja California: tendencias recientes", en Benjamín Burgos, Alejandro Mungaray y Juan Manuel Ocegueda (coords.), Estructura económica y demanda de educación superior en el noroeste de México, Miguel Ángel Porrúa-Anuies, México, pp. 11-46.

Ocegueda Hernández, Juan Manuel (2005), “Comercio y crecimiento económico en Baja California”, Investigación Económica, 64 (251), Universidad Nacional Autónoma de México, pp. 111-139.

Ocegueda Hernández, Juan Manuel (2006), “Especialización productiva y crecimiento económico en Baja California”, en Alejandro Mungaray y Juan Manuel Ocegueda (eds.), Estudios económicos sobre Baja California, Miguel Ángel Porrúa-UABC, México, pp.17-36.

Paschaloudis, Dimitris y S Alexiadis (2001), "Kaldorian approach to the economic growth of Greek Regions", Seoul Journal of Economics, 5, Institute of Economic Research, Seúl, 449-470. 
Pindyck, Robert y Daniel Rubinfeld (1998), Econometric models and econometric forecast, McGraw-Hill, Boston, MA.

Rivera Bátiz, Luis y Paul Romer (1991), "Economic integration and endogenous growth", The Quarterly Journal of Economics, 106 (2), MIT Press, Massachusetts, pp. 531-555.

Romer, Paul (1986), "Increasing Returns and Long-Run Growth", Journal of Political Economy, 94 (5), University of Chicago Press, Chicago, pp. 1002-1037.

Romer, Paul (1990), "Endogenous Technological Change”, Journal of Political Economy, 98 (5), University of Chicago Press, Chicago, pp. S71-S102.

Ros, Jaime (2001), "Diferencias internacionales en los niveles de ingreso y las tasas de crecimiento: modelos y evidencia empírica", documento preparado para el seminario La teoría del desarrollo en los albores del siglo xxI, Santiago de Chile, julio.

Smith, Adam (1984), Investigación sobre la naturaleza y causa de la rique$z a$ de las naciones, Fondo de Cultura Económica, México.

Shugan, Steven (1994), "Explanations for Service Growth", en Richard Oliver y Roland Rust (eds.), Service Quality, Sage Publications, Newbury Park, California, pp. 223-240.

Tugores, Juan (2002), Economía internacional. Globalización e integración regional, McGraw-Hill, Madrid.

Velázquez, Lorenia y Araceli Andablo (1997), "Características del sector servicios en Sonora (1975-1993)", Región y Sociedad, 8 (13-14), El Colegio de Sonora, México, pp. 5-37.

Verdoorn, Petrus J. (1949), "Fattori che regolano lo sviluppo della produttività del lavoro", L'Industria, 1, Società Editrice il Mulino, Bolonia, pp. 45-53.

White, Halbert (1980), "A heteroskedasticity-consistent covariance matrix estimator and a direct test for heteroskedasticity", Econometrica, 48 (4), The Econometric Society, Princeton, NJ, pp. 817-838. 
Recibido: 4 de mayo de 2009. Aceptado: 30 de septiembre de 2009.

Karla Susana Barrón-Arreola. Es doctora en ciencias económicas por la Universidad Autónoma de Baja California (UABC) y licenciada en economía por la Universidad Autónoma de Nayarit (UAN). Actualmente es miembro del Sistema Nacional de Investigadores (SNI), nivel I. Se desempeña como profesor-investigador de la Unidad Académica de Economía de la UAN y en el programa de maestría en desarrollo económico local como profesor del núcleo básico y responsable técnico del mismo. Entre sus publicaciones destacan: en coautoría, "Conformación de la microempresa marginada en la frontera norte de México", Estudios Fronterizos, 8 (15), uABC, Tijuana, pp. 51-72 (2007); "Microempresarialidad y pobreza en Baja California”, en I. Soloaga y G. Castañeda (coords.), Sobre México: temas actuales de politica económica, Universidad Popular Autónoma del Estado de Puebla, Puebla, pp. 111-121 (2009).

Jesús Antonio Madera-Pacheco. Es doctor por la Universidad de Córdoba, España; maestro en desarrollo regional por El Colegio de la Frontera Norte (Colef); y licenciado en economía por la Universidad Autónoma de Nayarit (UAN). Actualmente es investigador nivel I en el Sistema Nacional de Investigadores (SNI); y profesor en el área de ciencias sociales y humanidades de la UAN. Entre sus publicaciones destacan: "Los recovecos de la memoria y del saber ser campesino. Un acercamiento etnográfico al manejo de recursos entre pastores de Castril de la Peña, España", Péndulo: Papeles de Bastitania, 9, Granada, pp. 401-424 (2008); "Rasgando los silencios: pequeñas historias de careo con pastores y su ganado por Castril de la Peña”, AGER, Revista de Estudios sobre Despoblación y DesarroIlo Rural, 4, Universidad de Zaragoza, Zaragoza, pp.135-158 (2005); "El cultivo de tabaco en Nayarit: viejos esquemas de producción, diferentes repercusiones en la organización del trabajo", Convergencia, 31, Universidad Autónoma del Estado de México, Toluca, pp. 223-250 (2003). 